\title{
Return of research results from genomic biobanks: cost matters
}

\author{
Marianna J. Bledsoe, MA'1 Ellen Wright Clayton, MD, JD², Amy L. McGuire, JD, PhD², \\ William E. Grizzle, MD, PhD ${ }^{4}$ P. Pearl O'Rourke, MD $^{5}$ and Nikolajs Zeps BSc (Hons), PhD ${ }^{6,7}$
}

Return of individual research results (IRRs) to study participants has been, and continues to be, hotly debated. The role of biobanks in the return of research results had received little attention until the recent publication from Susan Wolf et al., ${ }^{1}$ which presents a detailed discussion and 10 recommendations regarding the return of results from biobanks. The authors propose that if reidentification of an individual participant (whom they also call a "contributor") is possible, the biobank, as the central hub in a biobank research system, should enable the system to (i) clarify the criteria for evaluating findings and develop a list of returnable findings; (ii) analyze a particular finding in relation to the aforementioned list/criteria; (iii) reidentify the individual participant/contributor; and (iv) recontact them to offer the finding. Wolf et al. ${ }^{1}$ propose that the biobank would bear the long-term responsibility for the return of incidental findings (IFs) and IRRs rather than the investigators themselves. These recommendations neither sufficiently reflect the wide variability in both the purpose and nature of biobanks nor do they take into account the associated costs to biobanks and the research system. Biobanks encompass disease-specific and healthy cohorts and range from small collections in individual laboratories to international collections involving many thousands of people. ${ }^{2}$

Delivering genetic information is costly and complex. This is true even in the clinic setting, which has the advantage of preexisting relationships and expectations between individuals and health-care providers. Extending the obligation to biobanks in the ways suggested by Wolf et al. ${ }^{1}$ would require significant financial investment and could place an unsustainable burden on many biobanks and researchers using biobank specimens and health information. The recommendations could have a negative impact on research by creating a disincentive for the establishment of biobanks, the distribution of samples and data, and subsequent research on those specimens and data. In addition, given the large number of biobanks, there would likely be considerable duplication of effort in the identification of lists of returnable findings as well as inconsistency in interpretation about when certain findings should be returned.
To illustrate the cost and practical implementation challenges, it is useful to consider some examples. ClinSeq, which has been cited as an example demonstrating that return of research results from biobanks need not be prohibitively costly or difficult, ${ }^{3}$ is unique in a number of ways that limits its generalizability. The purpose of ClinSeq (http://www.genome.gov/ClinSeq/) is to pilot large-scale medical sequencing in a clinical research setting. The researcher in this project has a direct, ongoing relationship with the participants/contributors, and one of the main aims of the study is to explore issues around return of research results. The primary study includes return of research results, genetic counseling, and retesting in a Clinical Laboratory Improvement Amendments-approved laboratory. Of note, ClinSeq does not involve the return of results generated from secondary research by other investigators, a central issue faced by many biobanks.

By contrast, let us consider a more common type of biobank, the National Institutes of Health Database of Genotypes and Phenotypes (dbGaP) (http://www.ncbi.nlm.nih.gov/gap), which relies on the submission of genotypic and phenotypic data from multiple primary researchers, distributes large amounts of data for secondary research analysis and has no direct, ongoing relationship with study participants/contributors. dbGaP currently contains genomic data from more than 300 studies and hundreds of thousands of participants/contributors. Genotypic and phenotypic datasets have been distributed for more than 3,500 approved projects. Although dbGaP does not collect or maintain identifiable information about participants/contributors, reidentification may be possible if the submitting investigator retained identifying information. Even if the biobank ( $\mathrm{dbGaP}$ ) plays only a coordinating role for the return of IFs and IRRs, the impact could be enormous. Using $\mathrm{dbGaP}$ as an example, whose responsibility would it be to "look" for IFs and IRRs that are returnable-the primary investigator, $\mathrm{dbGaP}$, the secondary researcher, or all of the above as all hold the genomic data? How would duplicative or even inconsistent results from analyses of data from the same participant/contributor be handled? Why should the biobank's responsibility be limited to genomic data when proteomic data, metabolomic data, and microbiome data

${ }^{1}$ Department of Veterans Affairs, Washington, DC, USA; ${ }^{2}$ Center for Biomedical Ethics and Society, Vanderbilt University, Nashville, Tennessee, USA; ${ }^{3}$ Center for Medical Ethics and Health Policy, Baylor College of Medicine, Houston, Texas, USA; ${ }^{4}$ University of Alabama at Birmingham, Birmingham, Alabama, USA; ${ }^{5}$ Partners HealthCare, Boston, Massachusetts, USA; ${ }^{6}$ St John of God HealthCare, Perth, Australia; ${ }^{7}$ School of Surgery and School of Pathology and Laboratory Medicine, The University of Western Australia, Crawley, Australia. Correspondence: Marianna J. Bledsoe (marianna.bledsoe@va.gov) 
may also become available and what would be the impact of extending the obligation to these kinds of data? Who would be responsible for interacting with the large number of IRBs that would likely be involved with the return of individual research findings? What costs would be required to set up the infrastructure needed to deal with the ever increasing number of studies and potentially relevant findings from those studies? What National Institutes of Health staff time would be needed to establish criteria for return of research results, routinely update those criteria, and communicate them to primary investigators who have the genomic data and secondary researchers who may be using those datasets? Who would pay for obtaining an additional sample from the participant and repeating the analysis in a Clinical Laboratory Improvement Amendments-approved laboratory? Who would pay for the return of individual findings if the primary study no longer has funding but a secondary analysis generates a finding that meets the criteria for returning it to the participant/contributor? Who would recontact participants/contributors if the primary investigator is no longer at the collection site? The need to ensure access to appropriate counseling would also need to be considered. If litigation for the consequences of providing IFs and IRRs is a possibility, additional costs may accrue to biobanks and their institutions, and such threats could seriously deter the establishment of many biobanks that are critical for scientific advancement.

Although the absolute number of results that could be considered "returnable" is uncertain, as new potentially relevant variants are identified, the number and the burdens and costs of returning results will likely increase. ${ }^{4}$ Biobanks that distribute specimens for a broad range of research would, in many cases, become burdened so quickly with managing the return of results that they could do little else than analyze IFs and IRRs. If one accepts that biobanks have an obligation to establish criteria for evaluating IFs and IRRs, analyze those findings, reidentify the participant and recontact them to offer the findings, would not this obligation also extend to all genomic research analysis (whether using specimens from a biobank or not)? If so, the costs and burdens to the research enterprise more broadly could be enormous and one cost to society could be inhibiting important research.

The magnitude of the costs that would be involved in the return of research results from some biobanks calls into question the validity of the extensive duties proposed by Wolf et al. ${ }^{1}$ Ossorio argues that the duty to return individual findings from repository research is limited. ${ }^{5}$ She asserts that the duty to help is typically specified narrowly such that helping is only a duty when it poses little or no risk or burden to the helper and does not interfere with the helper's legitimate aims. Ossorio further acknowledges that under current budgetary and personnel constraints, and with currently available information technology, routine return of individual findings from research using repository materials would constitute a substantial burden on the scientific enterprise and would seriously frustrate the aims of both scientists and specimen/data contributors. She suggests that "the duty-to-return issue should be analyzed as a conflict between (possibly) helping some contributors now and (possibly) helping a greater number of people who would benefit in the future from the knowledge produced by research." We agree, particularly given that the implementation of the recommendations would interfere with the aims of the biobank by changing the purpose of research biobanks from research to clinical care. We assert that this line of argument would limit the duty to return research results, particularly from secondary research analyses.

In summary, the approach suggested by Wolf et al. ${ }^{1}$ does not apply well to the broad range of biobanks that the recommendations are intended to address. Almost all biobanks would be affected, and the recommendations would pose enormous consequences for many biobanks and researchers. The requirements and associated costs may inhibit the establishment and operation of many biobanks as well as subsequent sharing and use of specimens and data by secondary researchers. We acknowledge that it may be important to return, in certain circumstances, findings that are analytically and clinically valid, have been confirmed in a Clinical Laboratory Improvement Amendments lab, and have serious consequences for participants, but the call for the routine evaluation and return of IFs and IRRs from all biobanks is not justified. In addition, the management of IFs and IRRs will be altered as genomic sequencing becomes routine medical practice.

Moreover, besides financial burden, the potential benefits and harms of returning research findings to research participants/contributors must also be considered. Given the complexity of these issues, policies for the return of research results from biobanks must be considered on a case-by-case basis, based on an analysis of the risks and benefits to research participants and society as a whole. Routine return of research results from biobanks may come at a cost to society of inhibiting important research needed to improve the health of individuals and the public.

\section{ACKNOWLEDGMENTS}

Preparation of this article was supported by the Department of Veterans Affairs, Veterans Health Administration, Office of Research and Development, Biomedical Laboratory Research and Development Service (M.J.B), St. John of God HealthCare (N.Z.) and the National Center for Advancing Translational Sciences of the National Institutes of Health under Award Number UL1 TR000445 (E.C.). The authors are funded by their affiliated institutions. The viewpoints expressed herein represent the authors' personal perspectives and should not be taken as representing the official viewpoint or position of the Department of Veterans Affairs or the National Institutes of Health. Thanks to Kristina Hill for her assistance in gathering data for the article.

\section{DISCLOSURE}

M.J.B. is a program manager for the VA biorepositories and biobanks and is a member of the International Society for Biological and Environmental Repositories (ISBER). N.Z. operates a cancer biobank at St John of God HealthCare within its Pathology Practice in the Bendat Family Comprehensive Cancer Centre. He is a member 
of ISBER and the Australasian Biospecimen Network Association. W.E.G. operates tumor banks as part of the Breast, Pancreatic and Cervical SPOREs at the University of Alabama at Birmingham and the Pulmonary Hypertension Breakthrough Initiative and prospective tissue repositories as part of the Cooperative Human Tissue Network and the Comprehensive Cancer Center and is a member of ISBER. He is a member of the Ethics Committee of the U54 grant, U54 MSM/TU/UAB Comprehensive Cancer Center Partnership. E.W.C. has long been involved in the creation, maintenance, and assessment of BioVU and has been studying ethical issues in genetics/genomics research for many years. She was part of the working group on biobanks convened by Professor Wolf but is not an author of its final document because she did not endorse its analysis and conclusions. A.L.M. and P.P.O. declared no conflict of interest.

\section{REFERENCES}

1. Wolf SM, Crock BN, Van Ness B, et al. Managing incidental findings and research results in genomic research involving biobanks and archived data sets. Genet Med 2012;14:361-384.

2. Bledsoe MJ, Grizzle WE, Clark BJ, Zeps N. Practical implementation issues and challenges for biobanks in the return of individual research results. Genet Med 2012;14:478-483.

3. Hayden EC. DNA donor rights affirmed. Nature 2012;483:387.

4. Cassa CA, Savage SK, Taylor PL, Green RC, McGuire AL, Mandl KD. Disclosing pathogenic genetic variants to research participants: quantifying an emerging ethical responsibility. Genome Res 2012;22:421-428.

5. Ossorio P. Taking aims seriously: repository research and limits on the duty to return individual research findings. Genet Med 2012;14:461-466. 